Andrea Heistinger, Elisabeth Kosnik, Gabriele Sorgo

Sorgsame Landwirtschaft

Neue Ökologie | Band 4 
Sorgsame Landwirtschaft. Sorgsame Forschung. Sorgsamer Druck.

Wie alle Forschung sind auch Bücher vergänglich und sollen wieder in einen StoffKreislauf eingehen können. Was Sie gerade in Händen halten, ist ein Cradle to Cradle@ und PurePrint $\odot$ Silver zertifiziertes Buch. Es enthält nur gesunde Substanzen - anders als herkömmliche gedruckte Bücher. Möglich machen dies die von der Druckerei gugler* in Melk entwickelten Druckverfahren. Die Papiersorten, Farben und Lacke sind ökologisch so optimiert, dass sie zu 100\% wiederverwendet werden. So könnten Sie dieses Buch kompostieren und als Dünger für Ihr Gemüse verwenden. Vorher bitte lesen und gerne weitergeben.

Dankeschön!

Andrea Heistinger (Dipl.-Ing.), geb. 1974, hat Landwirtschaft mit den Schwerpunkten Ökolandbau und Agrarsoziologie studiert. Ihre Forschungsschwerpunkte sind Innovationen im Ökologischen Landbau und Familiensoziologie, Systemische Beratung für Familienbetriebe und Organisationen. www.andreaheistinger.at

Elisabeth Kosnik (Mag. Dr. phil.), geb. 1983, Kulturanthropologin, war als Postdoc am Forschungsprojekt Bio Hoch Drei beteiligt. Die Schwerpunkte ihrer Forschung und Lehre an der Universität Graz sind ökonomische Anthropologie, Umweltanthropologie und Food Studies.

Gabriele Sorgo (PD Dr. phil.), geb. 1961, Kulturhistorikerin, lehrt Pädagogische und Historische Anthropologie an der Universität Innsbruck. Ihre Forschungsschwerpunkte sind Konsumtheorie, Gabenökonomien und Geschlechterforschung. 
Andrea Heistinger, Elisabeth Kosnik, Gabriele Sorgo

\section{Sorgsame Landwirtschaft}

Resiliente Praktiken im Ökologischen Landbau

[transcript] 
Diese Publikation wurde vom Land Steiermark, Referat Wissenschaft und Forschung, finanziert.

Das land

Steiermark

$\Rightarrow$ Wissenschaft und Forschung

\section{Bibliografische Information der Deutschen Nationalbibliothek}

Die Deutsche Nationalbibliothek verzeichnet diese Publikation in der Deutschen Nationalbibliografie; detaillierte bibliografische Daten sind im Internet über http://dnb.d-nb.de abrufbar.

\section{(1) (1) $\Theta \Theta$}

Dieses Werk ist lizenziert unter der Creative Commons Attribution-NonCommercial-NoDerivs 4.0 Lizenz (BY-NC-ND). Diese Lizenz erlaubt die private Nutzung, gestattet aber keine Bearbeitung und keine kommerzielle Nutzung. Weitere Informationen finden Sie unter https://creativecommons.org/licenses/by-nc-nd/4.o/deed.de

Um Genehmigungen für Adaptionen, Übersetzungen, Derivate oder Wiederverwendung zu kommerziellen Zwecken einzuholen, wenden Sie sich bitte an rights@transcript-publishing.com

Die Bedingungen der Creative-Commons-Lizenz gelten nur für Originalmaterial. Die Wiederverwendung von Material aus anderen Quellen (gekennzeichnet mit Quellenangabe) wie z.B. Schaubilder, Abbildungen, Fotos und Textauszüge erfordert ggf. weitere Nutzungsgenehmigungen durch den jeweiligen Rechteinhaber.

\section{(C) 2022 transcript Verlag, Bielefeld}

Umschlaggestaltung: Maria Arndt, Bielefeld

Umschlagabbildung: Andrea Heistinger

Druck: Gugler GmbH, Melk/Donau

Print-ISBN 978-3-8376-4898-0

PDF-ISBN 978-3-8394-4898-4

https://doi.org/10.14361/9783839448984

Buchreihen-ISSN: 2569-7900

Buchreihen-eISSN: 2703-1039

Cradle to cradle Certified ${ }^{\mathrm{TM}}$ Pureprint.

Innovated by gugler*

Gesund. Rückstandsfrei. Klimapositiv.

Besuchen Sie uns im Internet: https://www.transcript-verlag.de

Unsere aktuelle Vorschau finden Sie unter www.transcript-verlag.de/vorschaudownload 